\title{
DEHYDROZINGERONE ANALOGUES: REACTION OF $O$-ALKYL DERIVATIVES OF VANILLIN AND METHYL CYCLOPROPYL KETONE
}

\author{
Adrijana Burmudžija, Jovana Muškinja, Zoran Ratković* \\ University of Kragujevac, Faculty of Science, Department of Chemistry, \\ Radoja Domanovića 12, 34000 Kragujevac, Republic of Serbia \\ *Corresponding author; E-mail: wor@kg.ac.rs \\ (Received January 10, 2017; Accepted January 17, 2017)
}

\begin{abstract}
O-Alkyl vanillines and methyl cyclopropyl ketone reacts under ClaisenSchmidt conditions yielding corresponding enone derivatives, dehydrozingerone analogues with cyclopropane ring fragment, (E)-1-cyclopropyl-3-(4-alkoxy-3methoxyphenyl)prop-2-en-1-ones. All new compounds were well characterized by IR, ${ }^{1} \mathrm{H}$ and ${ }^{13} \mathrm{C}$ NMR spectroscopy and physical data.
\end{abstract}

Keywords: vanillin, enone system, dehydrozingerone, cyclopropyl.

\section{INTRODUCTION}

Chalcones and its derivatives exhibit various pharmacological and biological activities.

They show good antimicrobial (OPLETALOVA, 2000; YAYLI et al., 2006; TRIVEDI et al., 2008), antifungal (MOSTAHAR et al., 2007; LAHTCHEV et al., 2008; BAG et al., 2009), antioxidant (Vogel et al., 2008; SIVAKUMAR et al., 2010; VASIL'EV et al., 2010), antiprotozoal (LUNARDI et al., 2003), antitrichomonal (OYEDAPO et al., 2004), antimalarial (MOTTA et al., 2006; LiM et al., 2007; AWASTHI et al., 2009), anti-inflammatory (HERENCIA et al., 1998; ZHANG et al., 2010) and anticancer activity (ROMAGNOLI et al., 2008; ECHEVERRIA et al., 2009; SZLISZKA et al., 2009; ILANGO et al., 2010). It is well known that free phenolic group in aromatic ring at position 4- was key factor important for strong antibacterial activity of numerous natural products (CHEN et al., 1997; TSUKIYAMA et al., 2002), beside of nature, position and number of substituent on aromatic rings.

Ginger root is excellent source for many kinds of active compounds. From ginger extracts have been isolated dehydrozingerone $\mathbf{1}$, zingerone $\mathbf{2}$, gingerols $\mathbf{3}$, shogaols $\mathbf{4}$, paradols 5 and their derivatives, with expressed bioactivity, such as anticancer, antioxidant, antimicrobial, anti-inflammatory, antidiabetic, anti-allergic (NAKAMURA and YAMAMOTO, 1983; Dugasani et al., 2010; Semwal et al., 2015). Those compounds, similar to the chalcones, in their structure contains vanillin fragment. 
<smiles>COc1cc(/C=C/C(C)=O)ccc1O</smiles><smiles>COc1cc(CCC(C)=O)ccc1O</smiles><smiles>COc1cc(CCC(=O)CC(O)C(C)C)ccc1O</smiles><smiles>COc1cc(CCC(=O)/C=C/CCCCOc2ccccc2)ccc1O</smiles><smiles>COc1cc(CCC(=O)CCCCCCCOc2ccccc2)ccc1O</smiles>

Conjugate enone system which is presented in the dehydrozingerone is structurally different from chalcones; instead of the aryl group to the carbonyl is attached the methyl one. Their enone system could be easily transformed into some usable heterocyclic derivatives (ABDEL-RAHMAN et al., 2007; KALIRAJAN et al., 2009).

It is therefore, not surprising that many synthetic methods have been developed for the preparation of heterocycles starting from chalcone precursors that have been tested for their antimicrobial activities.

Knowing that cyclopropane ring is present in a huge number of natural isolated molecules (such as terpenes, fatty acids, alkaloids, steroids...) it is not a surprise that many of them show different biological activities, from enzyme inhibition of herpes proteases (PINTO et al., 1996; WITVROUW et al., 1999) to antibiotic, herbicidal, antitumor, and antiviral properties (BOGER et al., 2001; FAUST, 2001; YOSHIDA et al., 2004). Well known are chrysanthemic acid, pyrethrin and pyrethroid derivatives, compounds related to natural and synthetic insecticides, with good insecticidal activities (SAKAGUCHI et al., 1998; CONCELLON et al., 2007).

Starting from our previous results in dehydrozingerone derivatives transformation (RATKOVIĆ et al., 2016), we want to prepare some dehydrozingerone analogues with alicyclic cyclopropane ring fragment. The first step was alkylation of free phenolic group in vanillin $\mathbf{6}$ and second one was Claisen-Schmidt condensation of obtained $O$-alkyl derivatives 7a-f with methyl cyclopropyl ketone, Scheme 1. Compounds 8a-f, (E)-1-cyclopropyl-3-(4-alkoxy-3methoxyphenyl)prop-2-en-1-ones were synthesized. NMR).

All new products were characterized by their spectral data (IR, ${ }^{1} \mathrm{H}$ NMR and ${ }^{13} \mathrm{C}$ 


\section{MATERIALS AND METHODS}

\section{General remarks}

All starting chemicals were commercially available and used as received, except that the solvents were purified by distillation. Chromatographic separations were carried out using silica gel 60 (Merck, 230-400 mesh ASTM) whereas silica gel on Al plates, layer thickness $0.2 \mathrm{~mm}$ (Merck), was used for TLC. IR spectra were recorded on a Perkin-Elmer One FT-IR spectrometer with a $\mathrm{KBr}$ disc, $v$ in $\mathrm{cm} \mathrm{cm}^{-1}$; NMR spectra were recorded on a Varian Gemini $200 \mathrm{MHz}$ spectrometer $\left(200 \mathrm{MHz}\right.$ for ${ }^{1} \mathrm{H}$ and $50 \mathrm{MHz}$ for ${ }^{13} \mathrm{C}$ ), using $\mathrm{CDCl}_{3}$ as solvent and TMS as the internal standard. ${ }^{1} \mathrm{H}$ and ${ }^{13} \mathrm{C}$ NMR chemical shifts were reported in parts per million (ppm) and were referenced to the solvent peak; $\mathrm{CDCl}_{3}\left(7.26 \mathrm{ppm}\right.$ for ${ }^{1} \mathrm{H}$ and 76.90 ppm for ${ }^{13} \mathrm{C}$ ). Multiplicities are represented by s (singlet), d (doublet), $\mathrm{t}$ (triplet), q (quartet) and $\mathrm{m}$ (multiplet). Coupling constants $(J)$ are in Hertz $(\mathrm{Hz})$.

\section{Experimental procedure}

\section{Chemistry}

Starting from vanillin $\mathbf{6}$, set of its $O$-alkyl derivatives, compounds $7 \mathbf{a}-\mathbf{f}$, was prepared by alkylation of free phenolic group with corresponding alkyl halides, following the previously described literature procedures, (OYEDAPO et al., 2004; KATRITZKY et al., 2006; TATSUZAKI et $a l ., 2006)$. $O$-Alkyl vanillines and methyl cyclopropyl ketone reacts under basic conditions (Claisen-Schmidt condensation) yielding corresponding enone compounds, (E)-1cyclopropyl-3-(4-alkoxy-3-methoxyphenyl)prop-2-en-1-ones, 8a-f in good yield, Scheme 1. Compounds 8a-f was prepared following previously described procedure (FRINGUELLI et al., 1994, 1995) and to slightly modified procedure (HAMADA and SHARSHIRA, 2011).

Compounds 8a and $\mathbf{8 f}$ are known compounds and their chemical synthesis was published earlier (COGNACQ, 1978; KUMAR and DHAR, 1995), while compounds 8b-e are new compound and their structure and spectral data are given.

\section{Chemical synthesis}

2.1. General procedure for synthesis of (E)-1-cyclopropyl-3-(4-alkoxy-3methoxyphenyl)prop-2-en-1-one, $8 a-f$

$\mathrm{NaOH}(3 \mathrm{~g})$ was dissolved in $20 \mathrm{~mL}$ of water and cooled to $15^{\circ} \mathrm{C}$ and $15 \mathrm{~mL}$ of ethanol was added. Into a cold solution, with intensive stirring, methyl cyclopropyl ketone (10 mmol in $5 \mathrm{~mL}$ of ethanol) was added dropwise during $30 \mathrm{~min}$. Reaction mixture was kept at temperature under $20^{\circ} \mathrm{C}$. Corresponding $O$-alkyl vanillin $(10 \mathrm{mmol})$ was dissolved in a $10 \mathrm{~mL}$ of ethanol and added dropwise into a reaction mixture and stirred for next $6 \mathrm{~h}$. Then mixture is left overnight in refrigerator. Product crystallize from $60 \%$ ethanolic solution as white powder.

The separated solid was filtered, washed with water, dried and recrystallized from ethanol.

In some cases, products do not separate as solid. Following procedure was applied to isolate them: ethanol from reaction mixture was evaporated under reduced pressure while product remains as oily residue. Products were extracted with $\mathrm{CH}_{2} \mathrm{Cl}_{2}(3 \times 50 \mathrm{~mL})$; organic 
layer was washed with water and dried over anhydrous $\mathrm{Na}_{2} \mathrm{SO}_{4}$. Solvent was distilled off and products crystallize in some cases on standing or from ether.

\subsubsection{Synthesis of (E)-1-cyclopropyl-3-(3,4-dimethoxyphenyl)prop-2-en-1-one, 8 a}

Yield: $78.2 \%(1.81 \mathrm{~g})$; m.p. $89-90^{\circ} \mathrm{C}$

${ }^{1} \mathbf{H}$ NMR (CDCl 3$): 0.91-0.99(\mathrm{~m}, 2 \mathrm{H}), 1.12-1.19(\mathrm{~m}, 2 \mathrm{H}), 2.19-2.31(\mathrm{~m}, 1 \mathrm{H}), 3.92(\mathrm{~s}$, $3 \mathrm{H}), 3.93(\mathrm{~s}, 3 \mathrm{H}), 6.77(\mathrm{~d}, J=16 \mathrm{~Hz}, 1 \mathrm{H}), 6.88(\mathrm{~d}, J=8.3 \mathrm{~Hz}, 1 \mathrm{H}), 7.09-7.18(\mathrm{~m}, 2 \mathrm{H}), 7.58$ $(\mathrm{d}, J=16.2 \mathrm{~Hz}, 1 \mathrm{H}) ;{ }^{13} \mathbf{C} \mathbf{N M R}\left(\mathbf{C D C l}_{3}\right): 11,19.4,55.9,56.0,109.8,111.1,122.9,124.6$, 127.7, 142, 149.3, 151.2, 199.8; IR (KBr): 3435, 2969, 2938, 2838, 1639, 1621, 1597, 1513, $1467,1439,1425,1394,1269,1203,1164,1141,1020,985,910,875,812,802 \mathrm{~cm}^{-1}$

\subsubsection{Synthesis of (E)-1-cyclopropyl-3-(4-ethoxy-3-methoxyphenyl)prop-2-en-1-one, $8 \boldsymbol{b}$}

Yield: $76.8 \%$ (1.89 g); m.p. $77-78^{\circ} \mathrm{C}$

${ }^{1} \mathbf{H}$ NMR (CDCl$)$ ): 0.91-0.99 (m, 2H), 1.11-1.19 (m, 2H), 1.49 (t, $J=7$ Hz, 3H), 2.19$2.31(\mathrm{~m}, 1 \mathrm{H}), 3.91(\mathrm{~s}, 3 \mathrm{H}), 4.14(\mathrm{q}, J=7 \mathrm{~Hz}, 2 \mathrm{H}), 6.76(\mathrm{~d}, J=16 \mathrm{~Hz}, 1 \mathrm{H}), 6.87(\mathrm{~d}, J=8 \mathrm{~Hz}$, 1H), 7.10-7.15 (m, 2H), $7.57(\mathrm{~d}, J=16.2 \mathrm{~Hz}, 1 \mathrm{H}) ;{ }^{13} \mathbf{C}$ NMR (CDCl$): 10.9,14.6,19.3,55.9$, 64.3, 110.1, 112.2, 122.8, 124.4, 127.4, 142, 149.4, 150.6, 199.7; IR (KBr): 3435, 3011, 2981, 2923, 2874, 1645, 1620, 1596, 1512, 1471, 1425, 1392, 1314, 1265, 1231, 1204, 1177 , $1165,1149,1034,983,904,869,800 \mathrm{~cm}^{-1}$ one, $8 \mathrm{c}$

2.1.3. Synthesis of (E)-1-cyclopropyl-3-(3-methoxy-4-isopropoxyphenyl)prop-2-en-1-

Yield: $74.6 \%(1.94 \mathrm{~g})$; m.p. $56-57^{\circ} \mathrm{C}$

${ }^{1} \mathbf{H}$ NMR (CDCl$): 0.91-1.00(\mathrm{~m}, 1 \mathrm{H}), 1.12-1.19(\mathrm{~m}, 1 \mathrm{H}), 1.40(\mathrm{~d}, J=6 \mathrm{~Hz}, 6 \mathrm{H}), 2.17-$ $2.31(\mathrm{~m}, 1 \mathrm{H}), 3.89(\mathrm{~s}, 3 \mathrm{H}), 4.53-4.67(\mathrm{~m}, 1 \mathrm{H}), 6.76(\mathrm{~d}, J=16 \mathrm{~Hz}, 1 \mathrm{H}), 6.89(\mathrm{~d}, J=8.6 \mathrm{~Hz}$, 1H), 7.10-7.15 (m, 2H), $7.57(\mathrm{~d}, J=16 \mathrm{~Hz}, 1 \mathrm{H}) ;{ }^{13} \mathbf{C}$ NMR (CDCl $): 11,19.4,22,56,71.3$, 110.7, 114.6, 122.7, 124.5, 127.6, 142.1, 149.8, 150.4, 199.8; IR (KBr): 3435, 3004, 2983, $2938,1645,1595,1509,1461,1424,1395,1385,1315,1265,1232,1207,1165,1152,1112$, $1087,1034,985,946,906,874,820 \mathrm{~cm}^{-1}$ $8 d$

\subsubsection{Synthesis of (E)-1-cyclopropyl-3-(3-methoxy-4-propoxyphenyl)prop-2-en-1-one,}

Yield: $58.5 \%(1.52 \mathrm{~g})$; m.p. $61^{\circ} \mathrm{C}$

${ }^{1} \mathbf{H}$ NMR (CDCl $): 0.91-1.00(\mathrm{~m}, 1 \mathrm{H}), 1.05(\mathrm{t}, J=7.4 \mathrm{~Hz}, 3 \mathrm{H}), 1.12-1.19(\mathrm{~m}, 2 \mathrm{H})$, 1.79-1.89 (m, 2H), 2.19-2.31 (m, 1H), $3.91(\mathrm{~s}, 3 \mathrm{H}), 4.02(\mathrm{t}, J=6.8 \mathrm{~Hz}, 2 \mathrm{H}), 6.76(\mathrm{~d}, J=16$ $\mathrm{Hz}, 1 \mathrm{H}), 6.87(\mathrm{~d}, J=8.2 \mathrm{~Hz}, 1 \mathrm{H}), 7.10-7.16(\mathrm{~m}, 2 \mathrm{H}), 7.57(\mathrm{~d}, J=16.2 \mathrm{~Hz}, 1 \mathrm{H}) ;{ }^{13} \mathbf{C} \mathbf{~ N M R}$ (CDCl3): 10.3, 11, 11.1, 19.3, 56, 110.3, 112.4, 122.9, 124.4, 127.4, 142.1, 149.6, 150.9, 199.8; IR (KBr): 3436, 2961, 2946, 2932, 2871, 1671, 1606, 1595, 1510, 1466, 1424, 1390, $1261,1233,1196,1165,1143,1100,1090,1006,806 \mathrm{~cm}^{-1}$

2.1.5. Synthesis of (E)-1-cyclopropyl-3-(3-methoxy-4-buthoxyphenyl)prop-2-en-1-one, $8 e$

Yield: $58.5 \%$ (1.52 g); m.p. $56-57^{\circ} \mathrm{C}$

${ }^{1} \mathbf{H}$ NMR (CDCl $)$ : 0.91-1.07 (m, 2H), $0.98(\mathrm{t}, 3 \mathrm{H}, J=7.2 \mathrm{~Hz}), 1.13-1.28(\mathrm{~m}, 2 \mathrm{H})$, 1.41-1.59 (m, 2H), 1.77-1.92 (m, 2H), 2.19-2.31 (m, 1H), $3.90(\mathrm{~s}, 3 \mathrm{H}), 4.06(\mathrm{t}, J=6.8 \mathrm{~Hz}$, 2H), $6.76(\mathrm{~d}, J=16 \mathrm{~Hz}, 1 \mathrm{H}), 6.87(\mathrm{~d}, J=8 \mathrm{~Hz}, 1 \mathrm{H}), 7.10-7.15(\mathrm{~m}, 2 \mathrm{H}), 7.57(\mathrm{~d}, J=16.2 \mathrm{~Hz}$, $1 \mathrm{H}) ;{ }^{13} \mathbf{C}$ NMR (CDCl $\left.\mathbf{3}\right): 10.9,13.8,19.1,19.3,31.0,55.9,68.7,110.3,112.4,122.9,124.4$, 
127.4, 142.1, 149.5, 150.9, 199.8; IR (KBr): 2961, 2937, 2872, 1647, 1620, 1596, 1512, $1464,1425,1393,1261,1230,1178,1165,1143,1032,984,903,799 \mathrm{~cm}^{-1}$

$8 f$

2.1.6. Synthesis of (E)-1-cyclopropyl-3-(4-benzyloxy-3-methoxyphenyl)prop-2-en-1-one,

Yield: $80.2 \%$ (2.47 g); m.p. $110-111^{\circ} \mathrm{C}$

${ }^{1} \mathbf{H}$ NMR (CDCl $\left.\mathbf{3}\right): 0.89-0.99(\mathrm{~m}, 2 \mathrm{H}), 1.11-1.18(\mathrm{~m}, 2 \mathrm{H}), 2.17-2.29(\mathrm{~m}, 1 \mathrm{H}), 3.92(\mathrm{~s}$, $3 \mathrm{H}), 5.18(\mathrm{~s}, 2 \mathrm{H}), 6.75(\mathrm{~d}, J=16 \mathrm{~Hz}, 1 \mathrm{H}), 6.87(\mathrm{~d}, J=8.2 \mathrm{~Hz}, 1 \mathrm{H}), 7.04-7.11(\mathrm{~m}, 2 \mathrm{H}), 7.29-$ $7.46(\mathrm{~m}, 5 \mathrm{H}), 7.55(\mathrm{~d}, J=16 \mathrm{~Hz}, 1 \mathrm{H}) ;{ }^{13} \mathbf{C} \mathbf{~ N M R}\left(\mathbf{C D C l}_{3}\right): 11,19.4,56,70.8,110.4,113.5$, 122.7, 124.7, 127.2, 128, 128.6, 136.5, 141.9, 149.8, 150.3, 199.8; IR (KBr): 3436, 3064, 3038, 3000, 2906, 2864, 1636, 1596, 1511, 1468, 1450, 1398, 1349, 1315, 1269, 1230, 1204, $1167,1141,1085,1022,971,908,807,744,732,697,565 \mathrm{~cm}^{-1}$.

\section{RESULTS AND DISCUSSION}

Dehydrozingerone analogues, possessing cyclopropyl group attached to carbonyl, were synthesized under Claisen-Schmidt conditions, starting from $O$-alkylated vanillines and methyl cyclopropyl ketone, yielding corresponding enone compounds (E)-1-cyclopropyl-3-(4alkoxy-3-methoxyphenyl)prop-2-en-1-one, 8a-f in good yield, suitable for further transformation.

\section{References:}

[1] Abdel-Rahman, A.A.H., Abdel-Megied, A.E.S., Hawata, M.A.M., Kasem, E.R., SHABAAN, M.T. (2007): Synthesis and antimicrobial evaluation of some chalcones and their derived pyrazoles, pyrazolines, isoxazolines and 5,6-dihydropyrimidine-2-( $1 H$ thiones. Monatshefte 138: 889-897.

[2] Awasthi, S.K., Mishra, N., Kumar, B., Sharma, M., Bhattacharya, A., Mishra, L.C., BHASIN, V.K. (2009): Potent antimalarial activity of newly synthesized substituted chalcone analogs in vitro. Med. Chem. Res. 18: 407-420.

[3] Bag, S., Ramar S., Degani, M.S. (2009): Synthesis and biological evaluation of $\alpha, \beta$ unsaturated ketone as potential antifungal agents. Med. Chem. Res. 18: 309-316.

[4] Boger, D.L., Hughes, T.V., Hedrick, M.P., (2001): Synthesis, chemical properties, and biological evaluation of CC-1065 and Duocarmycin analogues incorporating the 5methoxycarbonyl-1,2,9,9a-tetrahydrocyclopropa[c]benz[e]indol-4-one alkylation subunit. J. Org. Chem. 66: 2207-2216.

[5] Chen, M., Christensen, S.B., Zhai, L., Rasmussen, M.H., Theander, T., Frokjaer, S., Steffansen, B., Davidsen, J., KharazMi, A. (1997): The novel oxygenated chalcone, 2,4-dimethoxy-4'-butoxychalcone, exhibits potent activity against human malaria parasite Plasmodium falciparum in vitro and rodent parasites Plasmodium berghei and Plasmodium yoelii in vivo. J. Infect. Dis. 176: 1327-1333.

[6] CognacQ, J-C. (1978): 1-(Aryloxy)-3-amino-2-propanols. Ger. Offen. DE 2736106 A1 19780223. 
[7] Concellon, J.M., RodrigueZ-Solla, H., Simal, C. (2007): The first cyclopropanation reaction of unmasked $\alpha, \beta$-unsaturated carboxylic acids: Direct and complete stereospecific synthesis of cyclopropanecarboxylic acids promoted by $\mathrm{Sm} / \mathrm{CHI}_{3}$. Org. lett. 9: 2685-2688.

[8] Dugasani, S., Pichika, M. R., Nadarajah, V.D., BalijePalli, M.K., Tandra, S., KORLAKUNTA, J.N. (2010): Comparative antioxidant and anti-inflammatory effects of [6]-gingerol, [8]-gingerol, [10]-gingerol and [6]-shogaol. J. Ethnopharmacol. 127: 515520.

[9] Echeverria, C., Santibanez, J.F., Donoso-Tauda, O., Escobar, C.A., Tagle, R.R. (2009): Structural antitumoral activity relationships of synthetic chalcones. Int. J. Mol. Sci. 10: 221-231.

[10] FAUST, R. (2001): Fascinating fatural and artificial cyclopropane architectures. Angew. Chem. Int. Ed. 40: 2251-2253.

[11] Fringuelli, F., Pani, G., Piermatti, O., Pizzo, F. (1994): Condensation reactions in water of active methylene compounds with arylaldehydes. One-pot synthesis of flavonols. Tatrahedron 50: 11499-11508.

[12] Fringuelli, F., Pani, G., Piermatti, O., Pizzo, F. (1995): Epoxidation of $\alpha, \beta$ unsaturated ketones in water. An environmentally benign protocol. Life Chem. Rep. 13: 133-140.

[13] Hamada, N.M.M., SharshiRa, E.M. (2011): Synthesis and antimicrobial evaluation of some heterocyclic chalcone derivatives. Molecules 16: 2304-2312.

[14] Herencia, F., Ferrandiz, M.L., Ubeda, A., Dominguez, J.N., Charris, E.J., Lobo, G.M., AlCaraz, M.J. (1998): Synthesis and anti-inflammatory activity of chalcone derivatives. Bioorg. Med. Chem. Lett. 8: 1169-1174.

[15] Ilango, K., Valentina, P., Saluja, G. (2010): Synthesis and in vitro anticancer activity of some substituted chalcones derivatives. Res. J. Pharm. Biol. Chem. Sci. 1: 354-359.

[16] Kalirajan, R., Sivakumar, S.U., Jubie, S.; Gowramma, B., Suresh, B. (2009): Synthesis and biological evaluation of some heterocyclic derivatives of chalcones. Int. J. ChemTech. Res. 1: 27-34.

[17] Katritzky, A.R., Long, Q., He, H., QIUA, G., WilcoX, A.L. (2006): Preparation of 2alkoxy-5-methoxybenzaldehydes and 2-ethoxy-5-alkoxybenzaldehydes. Arkivoc (vi): 868-875.

[18] Kumar, E.S., DHAR, D.N. (1995): Reaction of styryl cyclopropyl ketones with chlorosulfonyl isocyanate: Formation of iminosulfonyl chloride. Ind. J. Chem. 34B 1: 63-64.

[19] Lahtchev, K.L., Batovska, D.I., Parushev, S.P., Ubiyvovk, V.M., Sibirny, A.A. (2008): Antifungal activity of chalcones: a mechanistic study using various yeast strains. Eur. J. Med. Chem. 43: 2220-2228.

[20] LIM, S. S., KIM, H. S., LEE, D. U. (2007): In vitro antimalarial activity of flavonoids and chalcones, Bull. Korean Chem. Soc. 28: 2495-2497.

[21] Lunardi, F., Guzela, M., Rodrigues, A. T., Corre, R., Eger-Mangrich, I., Steindel, M., Grisard, E.C., Assreuy, J., Calixto, J.B., Santos, A.R.S. (2003): 
Trypanocidal and leishmanicidal properties of substitution-containing chalcones, Antimicrob. Agents Chemother. 47: 1449-1451.

[22] Mostahar, S., Katun, P., Islam, A. (2007): Synthesis of two vanillin ring containing flavones by different methods and studies of their antibacterial and antifungal activities. J. Biol. Sci. 7: 514-519.

[23] Motta, L.F., Gaudio, A.C., TaKahata, Y. (2006): Quantitative structure-activity relationships of a series of chalcone derivatives (1,3-diphenyl-2-propen-1-one) as antiPlasmodium falciparum agents (anti malaria agents). Internet Electron. J. Mol. Des. 5: 555-569.

[24] Nakamura, H., Yamamoto, T. (1983): The active part of the [6]-gingerol molecule in mutagenesis. Mutat. Res. Lett. 122: 87-94.

[25] Opletalova, V. (2000): Chalcones and their heterocyclic analogs as potential therapeutic agents in bacterial diseases. Cesk. Slov. Farm. 49: 278-284.

[26] Oyedapo, A.O., Mankanju, V.O., Adewunmi, C.O., Iwalewa, E.O., Adenowo, T.K. (2004): Antitrichomonal activity of 1,3-diaryl-2-propen-1-ones on Trichomonas gallinae. Afr. J. Trad. CAM 1: 55-62.

[27] Pinto, I.L., West, A., Debouckm, C.M., Dilella, A.G., GorniaK, J.G., O'Donnell, K.C., O'Shannessy, D.J., Patel, A., Jarvest, R.L. (1996): Novel, selective mechanism-based inhibitors of the herpes proteases. Bioorg. Med. Chem. Lett. 6: 2467-2472.

[28] Ratković, Z., MušKinja, J., Burmudžija, A., Ranković, B., Kosanić, M., Bogdanović, G. A., Simović-Marković, B., Nikolić, A., ArseniJević, N., ĐorĐevic, S., VukiĆEVIĆ, R.D. (2016): Dehydrozingerone based 1-acetyl-5-aryl-4,5-dihydro-1H-pyrazoles: Synthesis, characterization and anticancer activity. J. Mol. Struct. 1109: 82-88.

[29] Romagnoli, R., Baraldi, P.G., Carrion, M.D., Cara, C.L., Cruz-Lopez, O., Preti, D. (2008): Design, synthesis, and biological evaluation of thiophene analogues of chalcones. Bioorg. Med. Chem. 16: 5367-5376.

[30] Sakaguchi, K., Mano, H., Ohfune, Y. (1998): Syntheses of optically active 2substituted cyclopropanecarboxylic acids from chiral $\alpha$-hydroxysilane derivatives. Tetrahedron Lett. 39: 4311-4312.

[31] Semwal, R.B., Semwal, D.K., Combrinck, S., Viljoen, A.M. (2015): Gingerols and shogaols: Important nutraceutical principles from ginger. Phytochemistry 117: 554-568.

[32] Sivakumar, P.M., Prabhakar, P.K., Doble, M. (2010): Synthesis, antioxidant evaluation and quantitative structure activity relationship studies of chalcones. Med. Chem. Res. 19: 1-17.

[33] Szliszka, E., Czuba, Z.P., Mazur, B., Sedek, L., Paradysz, A., Krol, W. (2009): Chalcones enhance TRAIL-induced apoptosis in prostate cancer cells. Int. J. Mol. Sci. 11: $1-13$.

[34] Tatsuzaki, J., Bastow, K.F., Nakagawa-Goto, K., Nakamura, S., Itokawa, H., LEE, K. (2006): Dehydrozingerone, chalcone, and isoeugenol analogues as in vitro anticancer agents. J. Nat. Prod. 69: 1445-1449.

[35] Trivedi, A.R., Dodiya, D.K., Ravat, N.R., Shah, V.H. (2008) Synthesis and biological evaluation of some new pyrimidines via a novel chalcone series. Arkivoc (xi): 131-141. 
[36] Tsukiyama, R.I., Katsura, H., Tokuriki, N., Kobayashi, M. (2002): Antibacterial activity of licochalcone A against spore-forming bacteria. Antimicrob. Agents Chemother. 46: 1226-1230.

[37] Vasil'ev, R.F., Kancheva, V.D., Fedorova, G.F., Batovska, D.I., Trofimov, A.V. (2010): Antioxidant activity of chalcones: The chemiluminescence determination of the reactivity and the quantum chemical calculation of the energies and structures of reagents and intermediates. Kinet. Katal. 51: 507-515.

[38] Vogel, S., Ohmayer, S., Brunner, G., Heilmann, J. (2008): Natural and non-natural prenylated chalcones: Synthesis, cytotoxicity and antioxidative activity. Bioorg. Med. Chem. 16: 4286-4293.

[39] Witvrouw, M., Pannecouque, C., ClercQ, E.D., Fernandez-Alvarez, E., Marco, J.L. (1999): Inhibition of human immunodeficiency virus type (HIV-1) replication by some diversely functionalized spirocyclopropyl derivatives. Arch. Pharm. 332: 163166.

[40] Yayli, N., Ucuncu, O., Yasar, A., Kucuk, M., Akyuz, E, Karaoglu, S.A. (2006): Synthesis and biological activities of $N$-alkyl derivatives of $o-, m-$, and $p$-nitro $(E)-4-$ azachalcones and stereoselective photochemistry in solution with theoretical calculations. Turk. J. Chem. 30: 505-514.

[41] Yoshida, S., Rosen, T.C., Meyer, O.G.J., Sloan, M.J., Ye, S., Haufe, G., KiRK, K.L. (2004): Fluorinated phenylcyclopropylamines. Part 3: Inhibition of monoamine oxidase A and B. Bioorg. Med. Chem. 12: 2645-2652.

[42] Zhang, X.W., ZhaO, D.H., Quan, Y.C., Sun, L.P., Yin, X.M., Guan, L.P. (2010): Synthesis and evaluation of anti-inflammatory activity of substituted chalcone derivatives. Med. Chem. Res. 19: 403-412.

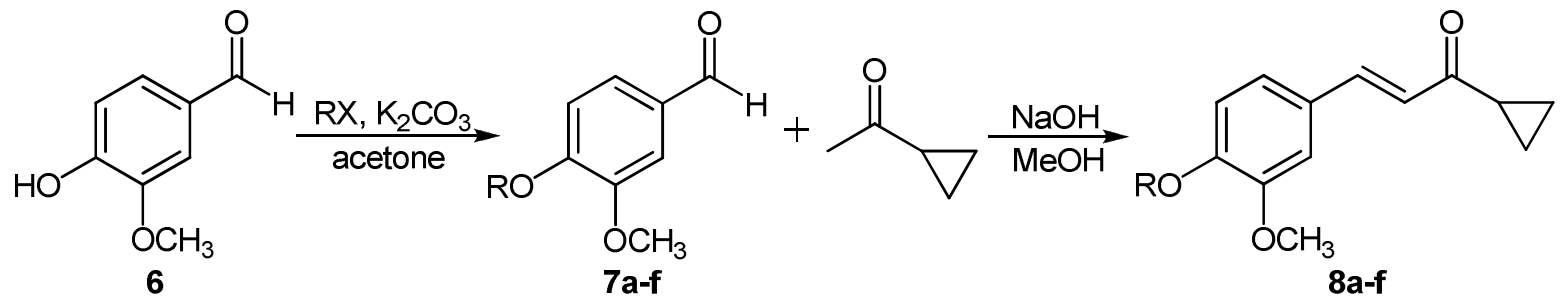

a) $\mathrm{R}=\mathrm{CH}_{3}$, b) $\mathrm{R}=\mathrm{C}_{2} \mathrm{H}_{5}$, c) $\mathrm{R}=i-\mathrm{C}_{3} \mathrm{H}_{7}$, d) $\left.\mathrm{R}=\mathrm{C}_{3} \mathrm{H}_{7}, \mathrm{e}\right) \mathrm{R}=\mathrm{C}_{4} \mathrm{H}_{9}$, f) $\mathrm{R}=\mathrm{C}_{6} \mathrm{H}_{5} \mathrm{CH}_{2}$

Scheme 1. Synthesis of (E)-1-cyclopropyl-3-(3-alkoxy-4-methoxyphenyl)prop-2-en-1-one, 